\title{
Morfometría de los Ramos del Nervio Plantar Lateral Antes de su División en Ramos Superficial y Profundo
}

\author{
Morphometry of the Branches of the Lateral Plantar Nerve Before \\ to its Division in Superficial and Deep Branches \\ "Mariano del Sol; Bélgica Vásquez \& Claudia Nicklas
}

DEL SOL, M.; VÁSQUEZ, B. \& NICKLAS, C. Morfometría de los ramos del nervio plantar lateral antes de su división en ramos superficial y profundo. Int. J. Morphol., 24(1):191-194, 2006.

RESUMEN: Los músculos del pie son frecuentemente afectados por accidentes que pueden comprometer su inervación. Así, el conocimiento anatómico de los nervios plantares y de sus ramos musculares adquiere importancia en los aspectos morfológico y quirúrgico. Se ha efectuado esta investigación debido a la poca información morfométrica existente respecto a los nervios de la musculatura inervada por el nervio plantar lateral, antes de su división en ramos superficial y profundo.

En 5 pies de cadáveres adultos, se disecó el nervio plantar lateral desde su origen hasta su división en ramos superficial y profundo identificándose los ramos originados en esta parte. A través de cortes histológicos transversales de los nerviosse obtuvo información cuantitativa sobre el número de fascículos, área de los fascículos y número de fibras por nervio.

El número promedio de fascículos de los nervios para los músculos abductor del dedo mínimo y cuadrado plantar, fue de 3 . El área promedio de los fascículos para los músculos abductor del dedo mínimo, cuadrado plantar y ramo cutáneo lateral, fue $0,165 \mathrm{~mm}^{2}$ (DE 0,119 ), $0,070 \mathrm{~mm}^{2}(\mathrm{DE} 0,042)$ y $0,041 \mathrm{~m}^{2}$ (DE 0,005), respectivamente. El número de fibras en los nervios para losmúsculos abuctor del dedo mínimo y cuadrado plantar y ramo cutáneo lateral, fue de 1.161 (DE 111), 419 (DE 136) y 445 (DE 97), respectivamente.

Con este trabajo se espera contribuir al conocimiento morfométrico de los nervios del pie sirviendo, además, como un apoyo anatómico a la microcirugía reparadora que se realiza en esa región.

PALABRAS CLAVE: Nervios del pie; Nervio plantar lateral; Morfometría.

\section{INTRODUCCIÓN}

La inervación de los músculos intrínsecos del pie ha sido descrita con más exactitud en las últimas décadas, debido al avance de nuevas técnicas quirúrgicas, menos invasivas y con mejores resultados terapéuticos.

El nervio plantar lateral, antes de su división en ramos superficial y profundo, inerva los músculos abductor del dedo mínimo y cuadrado plantar dando, además, ramos sensitivos al margen lateral del pie.

La inervación de los músculos abductor del dedo mínimo y cuadrado plantar ha sido estudiada por Bordelon, 1983; Baxter \& Thigpen, 1984; Baxter et al., 1989; del Sol \& Prates, 1987, 1988; del Sol et al., 2002; Lumsden, et al., 2003, entre otros. También, ha sido descrita la distribución de algunos ramos sensitivos del nervio plantar lateral antes de su división (del Sol \& Prates, 1990) y variaciones anatómicas del origen de los ramos calcáneos mediales (del Sol et al., 1992). Sin embargo, escasos estudios sobre morfometría de los nervios plantares pueden ser encontrados en la literatura (del Sol et al., 2005).

Conocer el número de fibras que se distribuyen en cada uno de los ramos de un nervio, adquiere importancia durante los procedimientos quirúrgicos, como también en las estimulaciones con propósitos terapéuticos. Con tal objetivo se planteó el estudio morfométrico de los ramos proximales del nervio plantar lateral antes de su división. 


\section{MATERIAL Y MÉTODO}

De 5 pies de cadáveres adultos, de sexo masculino, provenientes de la Facultad de Medicina de la Universidad de La Frontera, Temuco, Chile, obtuvimos trozos de cada uno de los ramos originados del nervio plantar lateral desde su origen hasta su división en ramos superficial y profundo, los cuales fueron procesados para su inclusión en paraplast. Realizamos cortes de $5 \mu \mathrm{m}$ de grosor, los cuales fueron montados en portaobjetos y teñidos con Hematoxilina-Eosina y Tricrómico de Masson.

Determinamos el número de fibras nerviosas de cada fascículo y el número de fascículos existente en cada nervio. Además, determinamos el área de los fascículos por planimetría, a través del conteo de puntos.

Los cortes fueron fotografiados utilizando un microscopio Carl Zeiss, Axiolab, con cámara MC 80 DX. Para la morfometría utilizamos un microscopio Olympus con retículo ocular $(1 \mathrm{~mm} / 0,01 \mathrm{~mm})$ calibrándolo con un retículo objetivo.

\section{RESULTADOS}

El patrón normal del nervio plantar lateral originaba los ramos para los músculos abductor del dedo mínimo (ramos sensitivo, posterior y anterior), ramo para el músculo cuadrado plantar (1 ramo) y ramo cutáneo lateral (un ramo).

El ramo sensitivo originado del nervio para el músculo abductor del dedo mínimo tenía en promedio 2 fascículos, al igual que el ramo posterior del mismo nervio. En cambio, el ramo anterior entraba al cuerpo muscular como un solo fascículo.

En promedio, fueron 3 fascículos los que tenía el ramo nervioso para el músculo cuadrado plantar y 4, en promedio, el número de fascículos presentes en el origen del nervio cutáneo lateral.

El área de los ramos nerviosos originados del nervio plantar lateral antes de su división en ramos superficial y profundo, se presenta en la Fig. 1.

El promedio del área de cada uno de los fascículos que componen los respectivos nervios, fue de $0,165 \mathrm{~mm}^{2}$ (DE 0,019); el área del ramo sensitivo del nervio abductor del dedo mínimo fue de $0,023 \mathrm{~mm}^{2}$ (DE 0,007$)$ y para los ramos posterior y anterior fue de $0,059 \mathrm{~mm}^{2}$ (DE 0,019$)$ y $0,079 \mathrm{~mm}^{2}$ (DE 0,009), respectivamente. El área promedio del nervio para elmúsculo cuadrado plantar fue de $0,070 \mathrm{~mm}^{2}(\mathrm{DE} 0,042)$ y para el ramo cutáneo lateral $0,045 \mathrm{~mm}^{2}(\mathrm{DE} 0,010)$, respectivamente.

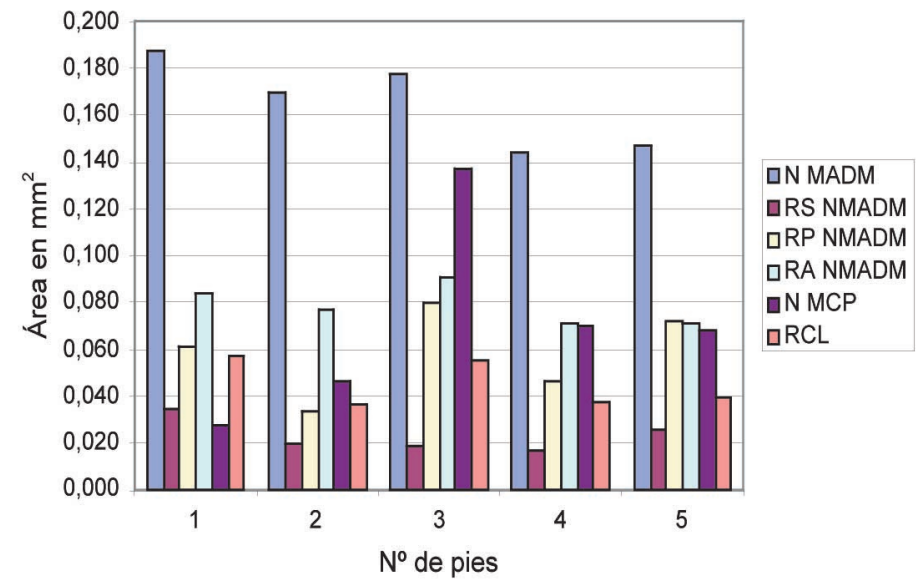

Fig. 1. Área de cada uno de los ramos originados del nervio plantar lateral antes de su división en ramos superficial y profundo.

El número de fibras totales en cada uno de los ramos nerviosos originados del nervio plantar lateral antes de su división en ramos superficial y profundo, se presenta en la Fig. 2.

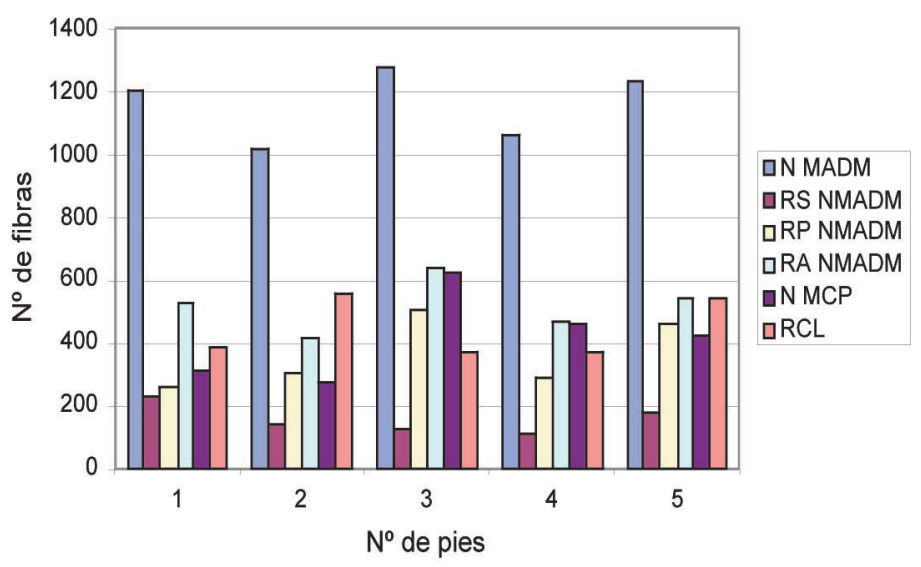

Fig. 2. Número de fibras en cada uno de los ramos originados del nervio plantar lateral antes de su división en ramos superficial y profundo.

El promedio del número de fibras que componen los respectivos nervios fue, de 1.161 (DE 111), del ramo sensitivo del nervio abductor del dedo mínimo fue de 159 (DE 47) y para los ramos posterior y anterior fue de 365 (DE 110) y 518 (DE 83), respectivamente. Para el músculo cuadrado plantar fue de 419 (DE 136) y para el ramo cutáneo lateral 445 (DE 97), respectivamente. 


\section{DISCUSIÓN}

El conocimiento anatómico y morfológico de la inervación de los músculos es cada vez más importante en la cirugía de la región, especialmente cuando se pretende obtener buenos resultados con abordajes menos radicales.

Se puede encontrar en los textos clásicos de las disciplinas morfológicas, el relato general del trayecto y las relaciones de los nervios espinales y menos específico de los nervios destinados a la musculatura del pie (Testut \& Latarjet, 1969; Rouvière \& Delmas, 1984; Sunderland, 1985; Williams et al., 1995 y Moore \& Dalley, 2002). Los trabajos de estos autores no abordan aspectos morfométricos.

Recientemente, del Sol et al. (2005) efectuaron un estudio de la morfometría de los nervios tibial, plantar lateral y plantar medial, abdordando aspectos morfométricos tales como el número de fibras nerviosas de cada fascículo y el número de fascículos existente en cada uno de estos nervios. Sin embargo, no hemos encontrado estudios morfométricos cuantitativos sobre los ramos musculares o sensitivos originados del nervio plantar lateral.

Del Sol \& Prates (1987) describieron la inervación del músculo cuadrado plantar y, un año después, del Sol \& Prates (1988) la inervación del músculo abductor del dedo mínimo. En estos trabajos no se adeterminó el número de fascículos y fibras, sino sólo aspectos mesoscópicos sobre el trayecto y las relaciones de cada uno de estos ramos emitidos por el nervio plantar lateral antes de su división en ramos superficial y profundo.

Según Baxter \& Thigpen, el dolor subcalcáneo de origen no traumático se debe a la compresión del nervio del músculo abductor del dedo mínimo por el espolón calcáneo. Para Bordelon, este ramo se dirige lateralmente, reposando en el ligamento plantar largo y dividiéndose en dos ramos, los que penetran en el músculo abductor del dedo mínimo, adyacente a la tuberosidad del calcáneo, hecho ratificado por del Sol et al. (2002), descartando así lo descrito por Przylucki \& Jones (1981), quienes describieron este ramo nervioso penetrando en el músculo a nivel del quinto metatarsiano. Este ramo corresponde al nervio cutáneo lateral, el cual se origina del nervio plantar lateral antes de su división (del Sol \& Prates 1990).

El fino ramo sensitivo originado del tronco del nervio para el músculo abductor del dedo mínimo, emite antes de su terminación en las proximidades del periostio del hueso calcáneo, frecuentemente, un ramo vascular que acompaña a los vasos destinados al músculo abductor del dedo mínimo, lo que explica la cantidad de fibras que lo componen.
Investigaciones realizadas por Lumsden et al. sobre la topografía distal del nervio tibial y de sus ramos, determinaron numerosas variaciones en cuanto a localización y trayecto de los ramos calcáneos mediales, hecho ya señalado por Soibelman, (1964) y del Sol \& Prates (1990). Así, al existir estas variaciones de origen de los ramos calcáneos mediales desde el nervio plantar lateral, el número de fascículos y de fibras nerviosas también van a ser variables.

De la misma manera, el número de fascículos y de fibras nerviosas que lleve el nervio plantar lateral como también cada uno de sus ramos, va a depender que se mantenga el padrón de normalidad, entre su origen y división. Sin embargo, existen numerosas variaciones entre las cuales se pueden mencionar, origen de nervios calcáneos mediales, presencia de un tronco común de los nervios para el músculo abductor del dedo mínimo y calcáneo medial, ramos vasculares, uno, dos o tres ramos para el músculo cuadrado plantar (es decir inervación separada para ambas cabezas del músculo) y la presencia de más de un ramo cutáneo lateral. Además, pueden encontrarse ramos para el músculo flexor corto del dedo mínimo, ramos anastomóticos para el nervio plantar medial y ramos para el ligamento calcáneo cuboideo plantar.

Nuestros resultados muestran que el área promedio de los fascículos en los nervios para el músculo abductor del dedo mínimo es bastante superior al área del músculo cuadrado plantar, ocurriendo lo mismo con el número de fibras presente en cada nervio. Esto posiblemente se debe a la acción más específica del $5^{\circ}$ dedo.

Cabe resaltar nuevamente la ausencia en la literatura de trabajos específicos sobre morfometría de los nervios destinados a la musculatura intrínseca del pie. Conocer aspectos como el perímetro de cada uno de uno de los nervios, el número de fibras nerviosas que los componen y las áreas de los fascículos, entre otros parámetros, es de interés morfológico ya que permite comparar la inervación del pie y de músculos homólogos. Resta por determinar estos mismos parámetros en cada uno de los nervios originados del ramo profundo del nervio plantar lateral, los cuales están destinados a la musculatura profunda de la planta del pie.

Finalmente, es posible afirmar que en la microcirugía reparadora se requiere de abdundante y detallada información anatómica y con este estudio esperamos haber contribuido a ello y, además, al conocimiento anatómico aportando nuevos antecedentes sobre la morfometría de los nervios de esta región del pie. 
DEL SOL, M.; VÁSQUEZ, B. \& NICKLAS, C. Morphometry of the branches of lateral plantar nerve, before to its division in superficial and deep branches. Int. J. Morphol., 24(1):191-194, 2006.

SUMMARY: Foot muscles are frequently affected by accidents that can involve their innervations. This is why the anatomy of the plantar nerves and the muscular branches become so important, in their morphological and chirurgic aspects. The reason underlying this research, is the insufficient information related to the nerves of the innerved muscles by the lateral plantar nerves, before to their division in superficial and deep branches.

Lateral plantar nerves were dissected, from their origin to their division in superficial and deep branches, identifying the originated branches in that part, in the feet of five adult corpses. With transversal histological nerve cuts, quantitative information is obtained, on fascicule number, fascicule area and fiber number per nerve.

The average number of the nerve fascicules for the abductor digiti minimi and quadratus plantae muscles was three. The average area of the nerve fascicules for the abductor digiti minimi and quadratus plantae muscles and lateral cutaneous branch was $0,165 \mathrm{~mm}^{2}$ (DE 0,119$)$, $0,070 \mathrm{~mm}^{2}$ (DE 0,042) and $0,041 \mathrm{~m}^{2}$ (DE 0,005). The number of fibers in the nerves for the abductor digiti minimi and quadratus plantae muscles and lateral cutaneous branch was 1.161 (DE 111), 419 (DE 136) y 445 (DE 97).

This research, is expected to contribute to the knowledge of the morphometry of the foot nerves and also become support for anatomists and reparatory microsurgery, carried out in this Region.

KEY WORDS: Nerves of the foot; Lateral plantar nerve; Morphometry.

\section{REFERENCIAS BIBLIOGRÁFICAS}

Baxter, D. E.; Pfeffer, G.B. \& Thigpen, C. M. Chronic heel pain:treatment rationale. Orthop. Clin. North. Am., 20:563-9, 1989.

Baxter, D. E. \& Thigpen, C. M. Heel pain:operative results. Foot Ankle, 5:16-25, 1984.

Bordelon, R. L. Subcalcaneal pain. Clin. Orthop., 177:49-53, 1983.

Del Sol, M. \& Prates, J. C. Inervação do músculo quadrado plantar no homem. Rev. bras. Ciências morfol., 4(1):20-3, 1987.

Del Sol, M. \& Prates, J. C. Inervação do músculo abductor digiti minimi do pé no homem. F. méd., 96(4):209-13, 1988.

Del Sol, M. \& Prates, J. C. Ramos cutâneos do nervo plantar lateral antes de sua divisão em ramos superficial e profundo (Ramo cutâneo lateral). Rev. IATROS, 6:63-5, 1990.

Del Sol, M.; Prates, J. C.; Olave, E. \& Ribeiro, E. Medial calcaneal branches from the lateral plantar nerve. Rev. Chil. Anat., 10(2):133-8, 1992.

Del Sol, M.; Olave, E.; Gabrielli, C.; Mandiola, E. \& Prates, J. C. Innervation of the Abductor digiti minimi muscle of the human foot. Anatomical basis of the entrapment of the Abductor digiti minimi nerve. Surg. radiologic. Anat., 24:18-22, 2002.

Del Sol, M.; Vasconcellos, A.; Parra, R. \& Vásquez, B. Morfometría de los nervios tibial y plantares. Int. J. Morphol., 23(4):399404, 2005.

Lumsden, D. B.; Schon, L. C.; Easley, M. E.; Duoiguih, W. A.; Anderson, C. D.; Miller, S. D. \& Ottey. D.K. Foot Ankle, 24(9):696-700, 2003.
Moore, K. L. \& Dalley, A. F. Anatomía con orientación clínica. $4^{\mathrm{a}}$ ed. Buenos Aires, Panamericana, 2002.

Przylucki, H. \& Jones, C. L. Entrapment neuropathy of muscle branch of lateral plantar nerve. J. Am. Podiatry Assoc., 71:11924, 1981.

Rouvière, H. \& Delmas, A. Anatomie humaine. $12^{\mathrm{a}}$ ed. Paris, Masson, 1984.

Soibelman, I. Observações anatômicas sôbre os rami calcanei mediales do nervo tibial no homem. Tesis de Doctorado, Escola Paulista de Medicina, São Paulo, 1964.

Sunderland, S. Nervios periféricos y sus lesiones. $2^{\mathrm{a}}$ ed. Barcelona, Salvat, 1985.

Testut, L. \& Latarjet, A. Tratado de Anatomía Humana. Salvat, Barcelona, 1969.

Williams, P.; Warwick, R.; Dyson, M. \& Bannister, L. Gray Anatomia. $37^{\mathrm{a}}$ ed. Rio de Janeiro, Guanabara Koogan, 1995.

Dirección para correspondencia:

Prof. Dr. Mariano del Sol

Facultad de Medicina

Universidad de La Frontera

Casilla 54-D

Temuco-CHILE

Email:mdelsol@ufro.cl

Recibido : 18-12-2005

Aceptado: 27-02-2006 